\title{
A novel amnion-chorion allograft membrane combined with a coronally advanced flap: a minimally invasive surgical therapy to regenerate interdental papillary soft tissue recession - a six-month postoperative image analysis-based clinical trial
}

\author{
Unnati Pitale ${ }^{1}$, Pritish Chandra Pal ${ }^{2}$, Ramanarayana Boyapati ${ }^{3}$, Ashish Bali ${ }^{2}$, Manish Varma ${ }^{4}$, Shaleen Khetarpal ${ }^{4}$ \\ ${ }^{1}$ Department of Periodontics, Modern Dental College \& Research Centre, Indore, \\ ${ }^{2}$ Department of Periodontics, Pacific Dental College and Hospital, Udaipur, \\ ${ }^{3}$ Department of Periodontology, Sibar Institute of Dental Sciences, Guntur, \\ ${ }^{4}$ Department of Periodontics, Govt. College of Dentistry, Indore, India
}

\begin{abstract}
J Korean Assoc Oral Maxillofac Surg 2021;47:438-444)
Objectives: Loss of the interdental papilla is multi-factorial and creates a multitude of problems. Autogenous connective tissue/biomaterial-based regeneration has been attempted for decades to reconstitute the black space created due to the loss of papilla. The aim of this present study was to regenerate papillary recession defects using an amnion-chorion membrane (ACM) allograft and to evaluate the clinical outcome up to six months postoperatively.

Materials and Methods: Twenty patients with 25 Nordland and Tarnow's Class I/II interdental papillary recession defects were treated with ACM and coronal advancement of the gingivo-papillary unit via a semilunar incision on the labial aspect followed by a sulcular incision in the area of interest. A photographic image analysis was carried out using the GNU Image Manipulation software program from the baseline to three and six months postoperatively. The black triangle height $(\mathrm{BTH})$ and the black triangle width (BTW) were calculated using the pixel size and were then converted into millimeters. The mean and standard deviation values were determined at baseline and then again at three and six months postoperatively. The probability values $(P<0.05$ and $P \leq 0.01)$ were considered statistically significant and highly significant, respectively. An analysis of variance and post hoc Bonferroni test were carried out to compare the mean values.

Results: Our evaluation of the BTH and BTW showed a statistically and highly significant difference from the baseline until both three and six months postoperatively $(P=0.01)$. A post hoc Bonferroni test disclosed a statistically significant variance from the baseline until three and six months postoperatively $(P<0.05)$ and a non-significant difference from three to six months after the procedure $(P \geq 0.05)$.

Conclusion: An ACM allograft in conjunction with a coronally advanced flap could be a suitable minimally invasive alternative for papillary regeneration.
\end{abstract}

Key words: Guided tissue regeneration, Gingival recession, Amnion, Chorion, Image analysis

[paper submitted 2021. 5. 8 / revised 1st 2021. 8. 31, 2nd 2021. 9. 15 / accepted 2021. 9. 23]

\section{Introduction}

The interdental papilla (IDP) represents the pyramidal shaped tissue formed by connective tissue covered by oral epithelium and has a complex morphological, anatomical,

\section{Pritish Chandra Pal}

Department of Periodontics, Pacific Dental College and Hospital, Hostel No. 2, Room No. 304, Debari, Udaipur 313024, India

TEL: +91-9977266593

E-mail: parthap634@gmail.com

ORCID: https://orcid.org/0000-0002-7654-7649

(c) This is an open-access article distributed under the terms of the Creative Commons Attribution Non-Commercial License (http://creativecommons.org/ licenses/by-nc/4.0/), which permits unrestricted non-commercial use, distribution, and reproduction in any medium, provided the original work is properly cited.

Copyright (C) 2021 The Korean Association of Oral and Maxillofacial Surgeons. and vascular structure. The combination of pristine gingiva and natural dentition is the best way to sustain oral health. Any deficiency of the papilla beneath the contact point of the teeth creates a black triangle that may lead to an increase in food and plaque accumulation and an inefficiency of selfcleansing action that may eventually progress to periodontal disease $^{1,2}$. The systemic interlink between general and oral health has already been proven; thus, the loss of IDP may negatively impact the overall health of an individual ${ }^{3}$. During the last decade, maintaining good oral health has become a major concern as well as a challenge in patients who require periodontal regenerative therapy. The regeneration of IDP especially in esthetic regions is among the most difficult treatment due to its anatomorphological situation and peculiarity 
of blood supply.

The existing literature reveals that several authors have tried different techniques to regenerate the IDP with discrete outcomes. Beagle ${ }^{4}$ reported on a surgical pedicle roll technique that was used to regenerate the lost IDP. Azzi et al. ${ }^{5}$ achieved surgical thickening of the gingiva and reconstruction of the IDP around an implant using a connective tissue graft (CTG). Kaushik et al. ${ }^{6}$ created an advanced papillary flap with interposed CTG that can be used to reconstruct the IDP if papillary loss occurs solely due to soft-tissue damage. Azzi's study ${ }^{7}$ reported gingival and papillary reconstruction using autogenous osseous and CTGs. In their case report, Carranza and Zogbi ${ }^{8}$ showed reconstruction of the IDP with an underlying CTG.

In the present scenario, regeneration is the key to the success of many treatments. Periodontal regeneration is now possible to a great extent with the use of advanced biomaterials like the amnion or chorion membrane ${ }^{9}$. Clinicians have used diverse periodontal biomaterials to reconstruct the IDP. Geurs et al. ${ }^{10}$ used a micronized acellular dermal graft in interproximal papillae regeneration. Gupta et al. ${ }^{11}$ suggested that both the amnion and chorion membranes are biocompatible and safe to use and do not cause any inadvertent tissue response or antigenic reactions. With the advancement of minimally invasive periodontal therapy, the novel human amnion-chorion membrane (ACM) tissue collected during Caesarean sections from the placenta has been widely utilized in the field of dentistry. ACM has showed successful gingival recession coverage, but its efficacy for papillary recession coverage is yet to be proven due to a lack of scientific research ${ }^{12}$.

Therefore, the aim of our present study was to regenerate Nordland and Tarnow's Class I and II papillary recession defects with the use of ACM allografts and also to evaluate the clinical outcomes up to six months postoperatively.

\section{Materials and Methods}

In this present multi-center, prospective in vivo clinical trial, ACM allografts were used to treat Class I or II papillary recession defects present in the maxillary anterior dentition ${ }^{13}$. Twenty total patients ( 9 males and 11 females) with 25 recession defect sites who were between 18 and 50 years of age (mean age, 30.96 years) were selected from our outpatient department. The Institutional Ethical Committee of the Modern Dental College and Research Center in Indore, India, approved the consent form and the experimental protocol (IRB No. MDCRC-2018-01-02-A) after a proper judgment. The study was performed within the time period of June 2019 to March 2020 and follow-up was completed by September 2020.

After the initial selection process, a comprehensive plaque control program was initiated three weeks before the procedure that included oral hygiene instruction, patient education, and motivation. All patients were in good health; they had a pocket depth $\leq 4 \mathrm{~mm}$, good plaque control, and tooth mobility scores of 0 . All patients with blood disorders, pregnant or lactating mothers, and tobacco users were excluded from the study population.

Informed written consent was obtained from the participants who fulfilled the inclusion criteria and voluntarily agreed to participate. A thorough explanation was given regarding the possible nature, risk, and benefit of the clinical investigations and the associated procedure ${ }^{3}$. The study was strictly performed according to the Declaration of Helsinki. Special care was taken to protect the confidentiality of the participants. A photographic image analysis was carried out preoperatively at baseline and then again at three and six months postoperatively and considered for statistical analysis. The ACM was purchased from the tissue bank of Tata Memorial Hospital in Mumbai. A tissue lab authority confirmed that the international protocol and standards were maintained from the time of tissue procurement until packaging. The safety of using the allograft was also ensured.

On the day of the surgery, local anesthesia was achieved by infiltrating the area with $2 \%$ lignocaine (Loxicard, lignocaine hydrochloride injection, $50 \mathrm{~mL}$; NEON, Chennai, India). The papillary recession height and width were measured with a periodontal probe on the buccal surface. The ACM allograft was cut to double the size of the papillary recession height and width. First, a semilunar incision approximately 6-10 $\mathrm{mm}$ apical from the gingival margin was placed on the labial aspect between the distolabial line angles of the involved teeth $^{14}$. Next, an intrasulcular incision was made around the mesial half of both the adjacent teeth to free the gingival attachment from the roots. An Orban interdental knife was then used to detach the gingivo-papillary unit from the underlying connective tissue to create a pouch-like space. The entire gingivo-papillary unit was also pushed coronally.(Fig. 1. A) The semilunar incision allows for coronal displacement without creating tension and prevents rebounding of the coronally advanced gingival position ${ }^{14}$. To maintain this new papillary position, the previously measured ACM allograft was then stuffed into the pouch space.(Fig. 1. B) Once the dry ACM came in contact with the blood present in the surgical field, it 

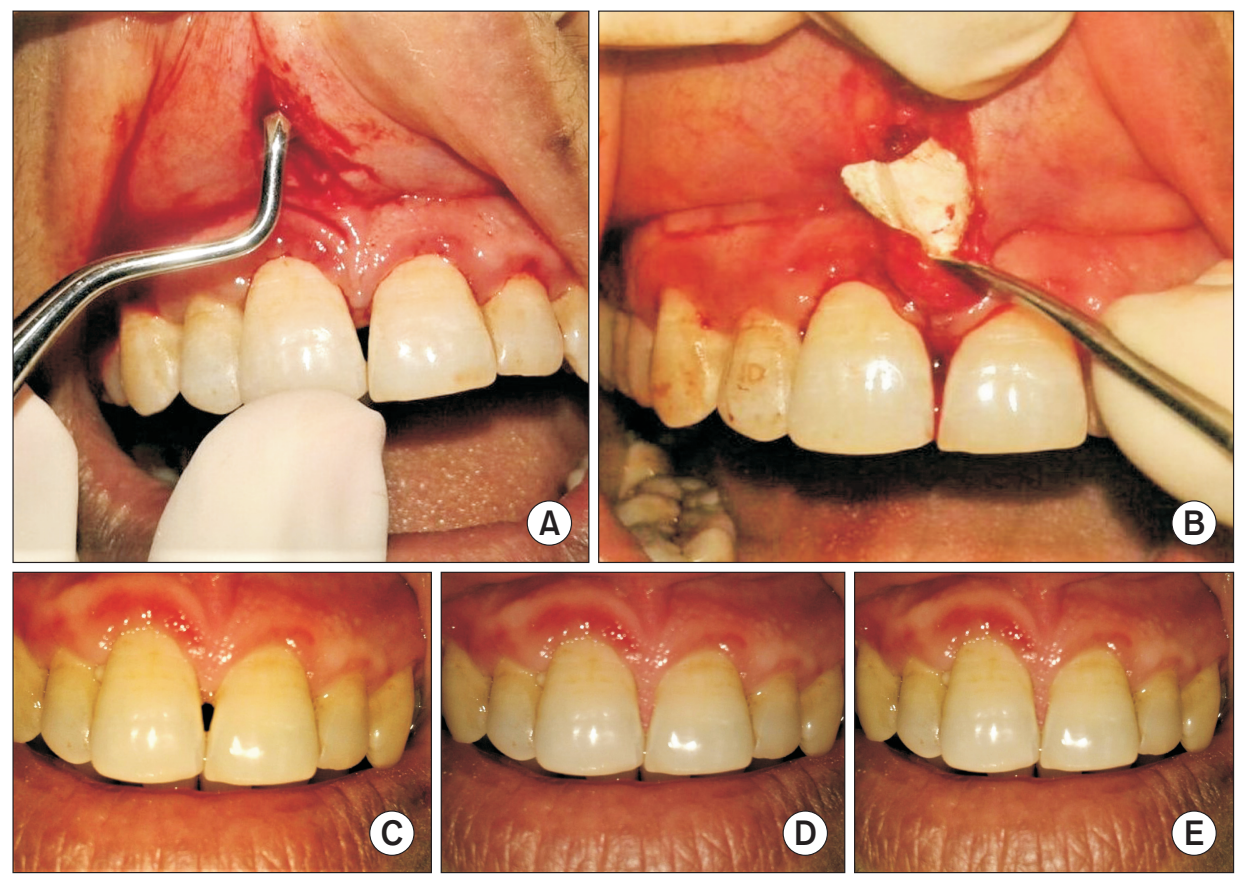

(E)
Fig. 1. A. The gingivo-papillary unit was coronally advanced to create a pouchlike space. B. The measured amnionchorion membrane allograft was placed into the pouch space. C. Photographs obtained at baseline show a papillary recession between \#11-\#21. D. The three-month postoperative result showing regeneration of the interdental papilla. E. The six-month evaluation showing persistent results.

Unnati Pitale et al: A novel amnion-chorion allograft membrane combined with a coronally advanced flap: a minimally invasive surgical therapy to regenerate interdental papillary soft tissue recession - a sixmonth postoperative image analysis-based clinical trial. J Korean Assoc Oral Maxillofac Surg 2021 was quickly hydrated and became supple. Then, the semilunar incision was sutured with interrupted 4-0 non-resorbable black silk sutures (Ethicon NW 3319 non-absorbable surgical suture; Johnson \& Johnson, Mumbai, India). Primary hemostasis was confirmed, and a periodontal dressing was placed. Fifteen days after surgery, suture removal was performed.

The patients were instructed to follow a soft, cold diet for three days postoperatively. They were prescribed analgesic antiinflammatory medication (Diclofenac sodium $50 \mathrm{mg}$, four times a day; Troikaa Pharmaceuticals, Ahmedabad, India) for three days. For persuasive plaque control, $10 \mathrm{~mL}$ of a $0.2 \%$ chlorhexidine mouth rinse (Chlohex ADS; Dr. Reddy's Laboratories, Hyderabad, India) was prescribed twice daily. A supportive periodontal therapy was performed weekly for the first four weeks and then monthly until the termination of the study period.

Photographs were taken (Nikon D5300 DSLR Camera; Nikon India, Gurgaon, India) at baseline (Fig. 1. C), three months postoperatively (Fig. 1. D), and six months postoperatively for evaluation.(Fig. 1. E) Paramount care was taken to maintain a similar photographic angulation and distance at the different follow-ups to allow a proper image analysis process (at a distance of $2 \mathrm{ft}$ from the patient, on macro mode, with the flash off, in natural sunlight, in autofocus mode). Once all the photographs were gathered, an image analysis was carried out using GNU Image Manipulation Program (GIMP) image analysis software, a free bitmap graphics editor. This software program calculates the number of tiny rectangular black pixels arranged in a grid formation, and the pixels are then transfigured into a physical length in millimeters. The entire process was done in GIMP by measuring the size of pixels of the reference object/line in the image. The difference between the maximum and minimum height was represented with " $y$ " coordinates within a designated area, while the width was shown using " $\mathrm{x}$ " coordinates that were prearranged by the computer program. The mesial to distal width of a central incisor was used as the reference distance. The wedge of the black triangle was judiciously measured and demarcated on the image. Then, the black triangle height (BTH) and the black triangle width (BTW) were automatically translated into a physical length $(\mathrm{mm})$. To attain the outcome, the contrast was enhanced in such a way that the dark portion on the photograph representing the deficient papillae showed up as completely black, while the remainder of the image was converted to white ${ }^{15}$.

The acquired data were processed through Microsoft Excel 2000 (Microsoft, Redmond, WA, USA), and the statistical analysis was carried out using the IBM SPSS Statistics for Windows (ver. 20.0; IBM, Armonk, NY, USA). The mean and standard deviation values for all the parameters were calculated at the baseline and then again at three and six months postoperatively. A $P<0.05$ was considered statistically significant, while $P \leq 0.01$ was considered highly significant. An analysis of variance test was conducted to evaluate the mean values of the BTH and BTW at the baseline and also again at three and six months postoperatively. A post hoc Bonferroni 

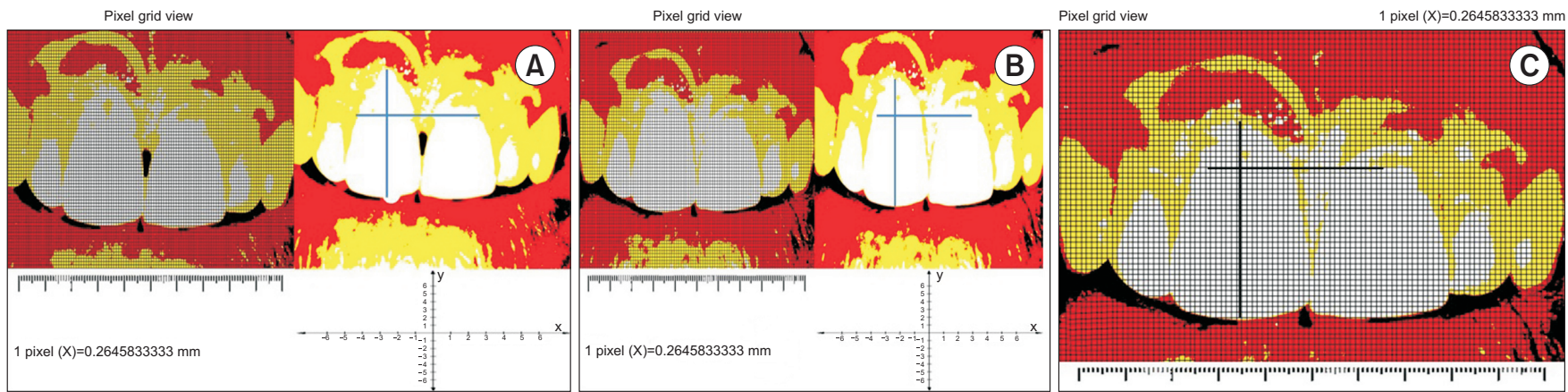

Fig. 2. A. An image analysis of papillary recession between \#11-\#21 at baseline. B. The image analysis shows an improvement of results after three months. C. The image analysis shows persistent results at six months postoperatively.

Unnati Pitale et al: A novel amnion-chorion allograft membrane combined with a coronally advanced flap: a minimally invasive surgical therapy to regenerate interdental papillary soft tissue recession - a six-month postoperative image analysis-based clinical trial. J Korean Assoc Oral Maxillofac Surg 2021

Table 1. The values of all the parameters measured at the baseline and then again at three-month and six-month intervals

\begin{tabular}{|c|c|c|c|c|}
\hline Parameter & Time interval & Mean \pm SD or mean difference $(\mathrm{mm})$ & F-value & $P$-value \\
\hline \multirow[t]{3}{*}{ BTH } & Baseline & $1.7920 \pm 0.93496$ & 13.413 & $0.01 *$ \\
\hline & $3 \mathrm{mo}$ & $0.7508 \pm 0.67027$ & & \\
\hline & $6 \mathrm{mo}$ & $0.8160 \pm 0.76067$ & & \\
\hline \multirow[t]{3}{*}{ BTW } & Baseline & $1.3525 \pm 0.66682$ & 13.601 & $0.01 *$ \\
\hline & $3 \mathrm{mo}$ & $0.5660 \pm 0.54262$ & & \\
\hline & $6 \mathrm{mo}$ & $0.5960 \pm 0.57475$ & & \\
\hline \multirow[t]{3}{*}{ BTH } & Baseline to $3 \mathrm{mo}$ & 1.04120 & & $0.01 *$ \\
\hline & Baseline to $6 \mathrm{mo}$ & 0.97600 & & $0.01 *$ \\
\hline & $3 \mathrm{mo}$ to $6 \mathrm{mo}$ & -0.06520 & & 0.955 \\
\hline \multirow[t]{3}{*}{ BTW } & Baseline to 3 mo & 0.78650 & & $0.01 *$ \\
\hline & Baseline to $6 \mathrm{mo}$ & 0.75650 & & $0.01 *$ \\
\hline & $3 \mathrm{mo}$ to $6 \mathrm{mo}$ & -0.03000 & & 0.983 \\
\hline
\end{tabular}

(SD: standard deviation, BTH: black triangle height, BTW: black triangle width)

$* P<0.05$.

Unnati Pitale et al: A novel amnion-chorion allograft membrane combined with a coronally advanced flap: a minimally invasive surgical therapy to regenerate interdental papillary soft tissue recession - a six-month postoperative image analysis-based clinical trial. J Korean Assoc Oral Maxillofac Surg 2021

test was conducted to uncover specific differences between the different timelines.

\section{Results}

The results of this study included a clinical as well as a photographic analysis-based augmentation of recession defects at three and six months postoperatively.(Fig. 2) A statistically highly significant difference was observed for both the BTH and the BTW at the three- and six-month followup assessments $(P=0.01)$.(Table 1) A post hoc Bonferroni test for parameters confirmed a statistically significant difference from the baseline to three and six months postoperatively $(P<0.05)$ and a non-significant difference from three to six months after surgery $(P \geq 0.05)$. (Table 1$)$ The image analysis method also concluded that $36 \%$ of sites ( 9 out of 25 sites) had achieved complete papillary coverage, while $64 \%$ of sites (16 out of 25 sites) had achieved a certain degree of partial coverage at three months. A further $28 \%$ of sites ( 7 out of 25 sites) remained as a complete papillary fill, and $72 \%$ of sites (18 out of 25 sites) had a varying degree of partial coverage at six months after the procedure. Measurements obtained using an image analysis process are more precise and can measure papillary loss $<1 \mathrm{~mm}$ or even a minute gain of soft tissue. The increasing availability, ease of operation, and improved quality of digital cameras has allowed their use to gain recent acceptance among clinicians for using digital images to assess minute details in clinical research.

\section{Discussion}

From the available literature, it is clear that the shape and health of the IDP are important for maintaining IDP hygiene via a self-cleansing mechanism; its absence may create problems in both esthetics and phonetics ${ }^{16,17}$. Together, these conditions can lead to a dearth of self-esteem; for individuals with a high lip line, it may become highly troublesome to the extent that the patient may avoid smiling due to a lack 
of self-confidence ${ }^{18}$. Thus, regeneration of lost IDP should be attempted in these individuals. Regeneration defines the re-growth of the lost tissues from the remaining portion. Although conventional autogenous tissue-based regenerative procedures may provide a good result, they also create a secondary donor site, which increases the morbidity associated with the procedure and also reduces patient acceptance. Regenerative medicine is a more modern approach that attempts to change the course of chronic diseases and tries to regenerate tissue in many ways.

Our present study attempted to regenerate missing tissue in Nordland and Tarnow's Class I or II papillary recession defects by combining an ACM allograft with a semilunar coronally advanced flap (CAF). The results of this study showed a statistically significant improvement in papillary augmentation from the baseline until three months and six months after the procedure was performed $(P<0.05)$. Further, 18 out of 25 recession sites achieved a stable result with complete papillary fill throughout the six-month study period.

Previous studies that have created a CAF in conjunction with autogenous CTG or bone grafts have reported a stable and predictable outcome $\mathrm{e}^{5-7}$. Nevertheless, the need to harvest an autograft unavoidably creates a secondary surgical site, which also increases the morbidity, surgical time, and risk of postoperative hemorrhaging or complications and therefore reduces a patient's willingness to undergo this procedure.

In contrast, evidence from increasing clinical use has proven the excellent efficiency of human placental allografts ${ }^{19}$. ACMs acquire antimicrobial and immune privilege properties that are conferred as a scaffold protein and thereby encourage cell migration to the area of interest. Current research has suggested that placental tissues are a good source of mesenchymal stromal cells ${ }^{20}$.

Novel ACM and placental allograft membranes are primarily a cryo-preserved and dehydrated laminate of amnion-chorion ( $\mathrm{dHACM}$ ). The unique distinctive properties of ACMs incorporate the presence of laminin-5 and growth factors like platelet-derived growth factor, fibroblast growth factor, and transforming growth factor beta ${ }^{21}$. It has been speculated that these proteins aid in rapid granulation over the allograft. dHACM demonstrates an enhanced growth factor expression profile and stromal derived factor 1 expression, which triggers inhabitant cells. ACM is therefore an appealing therapeutic modality because its structural component provides a scaffold for regeneration. The recruited cells show selfrenewal and differentiation properties and are localized near the sites of neovascularization, which is condemnatory for regeneration ${ }^{22}$.

ACM allografts have been extensively used to heal ophthalmic surfaces, burn sites, and diabetic foot ulcers. The amniotic extracellular substance is beneficial in regenerating peripheral nerves. De-cellularized amnion-chorion can be used as a nourishing layer for stem cells obligatory for neuronal differentiation ${ }^{23}$. ACM has been successfully used to treat chronic cutaneous wounds and exhibits a low immunogenicity and a reduction in inflammation and pain ${ }^{24}$. The exact mechanism of action of fetal membranes' clinical benefits has yet to be fully determined ${ }^{22}$.

In recent years, ACM has also been studied for periodontal regeneration and has shown promising results. Studies have been conducted using either amnion or chorion or as the ACM membrane to treat gingival recession, periodontal intrabony defects, and furcation areas with or without bone grafts. To the best of our knowledge, no clinical study has investigated using ACM as a gingival papillary regenerative material, although researchers have achieved successful soft tissue gains in various clinical situations ${ }^{12}$. Brain observed an increase in new gingival tissue that represented $97 \% \pm 0.5 \%$ root coverage when ACM was used in a moderate recession defect $^{25}$. Chakraborthy et al. ${ }^{12}$ found a mean decrease in the length of recession with a chorion membrane of $2.00 \pm 1.54$ $\mathrm{mm}$ and an amnion membrane of $1.58 \pm 1.14 \mathrm{~mm}$. Suresh and Gupta $^{26}$ reported a statistically significant gain of relative clinical attachment $(P=0.01)$ and a highly significant reduction of marginal gingival position ( $P=0.001)$ six months after creating an adjoining CAF using an amnion membrane in Millers Class I or II gingival recession ${ }^{11}$. When used in conjunction with chorion membrane, CAF also showed a similar and highly statistically significant mean gain in the clinical attachment level $(P=0.001)$ and a significant mean reduction in the gingival margin $(P=0.03)$ at the six-month follow-up examination $^{26}$. Suresh and Gupta ${ }^{26}$ achieved $100 \%$ root coverage and additional enhancement of the soft tissue biotype when ACM was used with CAF; these findings were also supported by reports published by Joshi et al. ${ }^{27}$. The choice of flap and selection of biomaterial for recession coverage greatly depends upon the clinical situation, such as the degree and stages of recession, along with the preference of the operator and patients. Evidence from past studies that have examined cases of gingival recession coverage with ACM has greatly reduced the surgical morbidity associated with this procedure by eliminating the need for a second surgical site and has also enhanced the acceptance of the treatment by avoiding any further psychological trauma. 
The aforementioned clinical studies clearly illustrate the tremendous potential of ACM in the field of soft tissue regenerative therapy. It was a wise option to combine ACM with a CAF to treat mild to moderate papillary recession. One possible explanation for the successful papillary fill with ACM when placed beneath a CAF could be explained by the fact that it acts as a potential source of stem cells and growth factors and enhances neo-angiogenesis. The potential of ACM to self-adhere eliminates the need for suturing ${ }^{28}$. An in vitro study revealed that ACM allografts combat degradation and perpetuate their physical form for up to three weeks. Periodontal regeneration is chiefly completed within two to three weeks with primary wound closure and is followed by a phase of remodeling and maturation before finally reaching its functional stipulation. Therefore, the use of fetal allograft ACM for regeneration also may offer present papillary coverage because the ACM can retain its physical form in the surgically created pouch space for a period of two to three weeks. Pollard et al. ${ }^{29}$ stated that ACM resists early degradation because it contains interstitial collagen.

The widespread use of ACM has been restricted by concerns with its source along with difficulties in preparation and storage and also the potential for infectious disease transmission. Current developments in tissue processing and establishing an international protocol have addressed these ethical issues. Fetal membranes obtained during Caesarean section deliveries are thoroughly washed in a sodium chloride isotonic medical solution. The amniotic-chorion is then gently separated and slit into sections. It is thoroughly washed sequentially in $0.5 \mathrm{M}, 1.0 \mathrm{M}$, and $1.5 \mathrm{M}$ solutions of dimethyl sulfoxide and is finally stored at $-80^{\circ} \mathrm{C}$. Since the $1980 \mathrm{~s}$, a series of research on ACM has failed to detect human leukocyte antigen or $\beta_{2}$-microglobulin in cultured amniotic epithelium, which led to its reported safety ${ }^{30}$.

Thus, within the limitations of our study, we conclude that $\mathrm{ACM}$ can be used in combination with a semilunar CAF technique as an alternative novel papillary regenerative therapy.

The minimally invasive reconstruction of the IDP with an allograft offers new hope for eliminating the black triangle. The successful use of this procedure may benefit both patients and clinicians in terms of causing minimal trauma and offering an easier or comparatively simpler procedure than an autogenous soft tissue graft. In the future, papillary regeneration around dental implants may significantly benefit from this concept of alternative material-based reconstruction.

\section{Conclusion}

The growing cosmetic demand from patients has solidified the importance of gingival esthetics. An intact IDP plays a prime role in maintaining a holistic esthetic appearance. Our current study showed that the ACM along with coronal advancement of the interdental unit had an enhanced beneficial effect on the regeneration of lost interdental papillary tissue. In addition, proper patient selection, surgical skills, and an adequate maintenance protocol are also crucial for successful outcomes.

\section{ORCID}

Unnati Pitale, https://orcid.org/0000-0003-2492-4286

Pritish Chandra Pal, https://orcid.org/0000-0002-7654-7649

Ramanarayana Boyapati, https://orcid.org/0000-00029196-0183

Ashish Bali, https://orcid.org/0000-0003-1618-3959

Manish Varma, https://orcid.org/0000-0002-0438-7586

Shaleen Khetarpal, https://orcid.org/0000-0003-1437-4241

\section{Authors' Contributions}

All authors contributed equally to this work. U.P., P.C.P., R.B., and M.V. participated in the study design, clinical procedure, data collection, data analysis and primary preparation of manuscript. A.B. and S.K. performed the image analysis, statistical analysis, wrote the manuscript and helped to draft the manuscript. All authors read and approved the final manuscript.

\section{Acknowledgements}

We would like to thank the department head of the Tissue Lab at Tata Memorial Hospital in Mumbai for providing us with ACM for this research. We would also like to thank the department head of the Oral Radiology, Modern Dental College and Research Center in Indore for guiding us with our image analysis.

\section{Ethics Approval and Consent to Participate}

This study was approved by the Institutional Ethical Review Board of the Modern Dental College and Research Center in Indore, India (IRB No. MDCRC-2018-01-02A), and written informed consent was approved by the IRB 


\section{Consent for Publishing Photographs}

Written informed consent was obtained from the patients for publication of this article and accompanying images.

\section{Conflict of Interest}

No potential conflict of interest relevant to this article was reported.

\section{References}

1. Singh VP, Uppoor AS, Nayak DG, Shah D. Black triangle dilemma and its management in esthetic dentistry. Dent Res J (Isfahan) 2013;10:296-301.

2. Shenoy BS, Punj A, Ramesh A, Talwar A. Salvaging the lost pink triangle: a case series of papilla reconstruction. Case Rep Dent 2020;2020:9735074. https://doi.org/10.1155/2020/9735074

3. Pitale U, Pal PC, Thakare G, Verma M, Dhakad S, Pandey R. Minimally invasive therapy for reconstruction of lost interdental papilla by using injectable hyaluronic acid filler. J Indian Soc Periodontol 2021;25:22-8. https://doi.org/10.4103/jisp.jisp_19_20

4. Beagle JR. Surgical reconstruction of the interdental papilla: case report. Int J Periodontics Restorative Dent 1992;12:145-51.

5. Azzi R, Etienne D, Takei H, Fenech P. Surgical thickening of the existing gingiva and reconstruction of interdental papillae around implant. Int J periodontol Rest Dent 2002;22:71-7.

6. Kaushik A, Pk P, Jhamb K, Chopra D, Chaurasia VR, Masamatti VS, et al. Clinical evaluation of papilla reconstruction using subepithelial connective tissue graft. J Clin Diagn Res 2014;8:ZC7781. https://doi.org/7860/JCDR/2014/9458.4881

7. Azzi R, Takei HH, Etienne D, Carranza FA. Root coverage and papilla reconstruction using autogenous osseous and connective tissue grafts. Int J Periodontics Restorative Dent 2001;21:141-7.

8. Carranza N, Zogbi C. Reconstruction of the interdental papilla with an underlying subepithelial connective tissue graft: technical considerations and case reports. Int J Periodontics Restorative Dent 2011;31:e45-50.

9. Ausenda F, Rasperini G, Acunzo R, Gorbunkova A, Pagni G. New perspectives in the use of biomaterials for periodontal regeneration. Materials (Basel) 2019;12:2197. https://doi.org/10.3390/ ma12132197

10. Geurs NC, Romanos AH, Vassilopoulos PJ, Reddy MS. Efficacy of micronized acellular dermal graft for use in interproximal papillae regeneration. Int J Periodontics Restorative Dent 2012;32:49-58.

11. Gupta A, Kediege S, Mittal A, Jain K, Gupta S, Chaudhry S. Amnion and chorion membranes in the treatment of gingival recession and their effect on gingival biotype: a clinical and ultrasonographic study. J Clin Diagn Res 2018;12:ZC26-32. https://doi.org/10.7860/ JCDR/2018/27765.11307

12. Chakraborthy S, Sambashivaiah S, Kulal R, Bilchodmath S. Amnion and chorion allografts in combination with coronally advanced flap in the treatment of gingival recession: a clinical study. J Clin Diagn Res 2015;9:ZC98-101. https://doi.org/10.7860/ JCDR/2015/12971.6572

13. Nordland WP, Tarnow DP. A classification system for loss of papillary height. J Periodontol 1998;69:1124-6. https://doi.org/10.1902/ jop.1998.69.10.1124

14. Han TJ, Takei HH. Progress in gingival papilla reconstruction. Periodontol 2000 1996;11:65-8. https://doi.org/10.1111/j.16000757.1996.tb00184.x
15. Bhattacharya S. Clinical photography and our responsibilities. Indian J Plast Surg 2014;47:277-80. https://doi.org/10.4103/09700358.146569

16. Holmes CH. Morphology of the interdental papillae. J Periodontol 1965;36:455-60. https://doi.org/10.1902/jop.1965.36.6.455

17. Cohen B. Pathology of the interdental tissues. Dent Pract 1959;9: 167-73.

18. Schroeder HE, Listgarten MA. The gingival tissues: the architecture of periodontal protection. Periodontol 2000 1997;13:91-120. https://doi.org/10.1111/j.1600-0757.1997.tb00097.x

19. Bourne G. The foetal membranes. A review of the anatomy of normal amnion and chorion and some aspects of their function. Postgrad Med J 1962;38:193-201. https://doi.org/10.1136/ pgmj.38.438.193

20. Koob TJ, Rennert R, Zabek N, Massee M, Lim JJ, Temenoff JS, et al. Biological properties of dehydrated human amnion/chorion composite graft: implications for chronic wound healing. Int Wound J 2013;10:493-500. https://doi.org/10.1111/iwj.12140

21. Lei J, Priddy LB, Lim JJ, Koob TJ. Dehydrated human amnion/ chorion membrane (dHACM) allografts as a therapy for orthopedic tissue repair. Tech Orthop 2017;32:149-57. https://doi.org/10.1097/ BTO.0000000000000229

22. Maan ZN, Rennert RC, Koob TJ, Januszyk M, Li WW, Gurtner GC. Cell recruitment by amnion chorion grafts promotes neovascularization. J Surg Res 2015;193:953-62. https://doi.org/10.1016/ j.jss.2014.08.045

23. Grueterich M, Tseng SC. Human limbal progenitor cells expanded on intact amniotic membrane ex vivo. Arch Ophthalmol 2002;120:783-90. https://doi.org/10.1001/archopht.120.6.783

24. Kjaergaard N, Hein M, Hyttel L, Helmig RB, Schønheyder HC, Uldbjerg N, et al. Antibacterial properties of human amnion and chorion in vitro. Eur J Obstet Gynecol Reprod Biol 2001;94:224-9. https://doi.org/10.1016/s0301-2115(00)00345-6

25. Gurinsky B. A novel dehydrated amnion allograft for use in the treatment of gingival recession: an observational case series. J Implant Adv Clin Dent 2009;1:65-73.

26. Suresh DK, Gupta A. Gingival biotype enhancement and root coverage using human placental chorion membrane. Clin Adv Periodontics 2013;3:237-42. https://doi.org/10.1902/cap.2012.120039

27. Joshi CP, Panjwani AA, D'Lima CB, Dani NH. Comparative evaluation of amnion-chorion membrane and chorion membrane for root coverage and gingival biotype enhancement: a case report. EC Dent Sci 2017;14:255-9.

28. Elzanaty M, Shoieb M, Ghallab NA, Nahass HEE. Clinical evaluation of amnion chorion membrane in comparison to subepithelial connective tissue graft in gingival recession coverage. Egypt Dent J 2017;30

29. Pollard SM, Aye NN, Symonds EM. Scanning electron microscope appearances of normal human amnion and umbilical cord at term. Br J Obstet Gynaecol 1976;83:470-7. https://doi.org/10.1111/ j.1471-0528.1976.tb00868.x

30. Stock SJ, Kelly RW, Riley SC, Calder AA. Natural antimicrobial production by the amnion. Am J Obstet Gynecol 2007;196:255.e16. https://doi.org/10.1016/j.ajog.2006.10.908

How to cite this article: Pitale U, Pal PC, Boyapati R, Bali A, Varma M, Khetarpal S. A novel amnion-chorion allograft membrane combined with a coronally advanced flap: a minimally invasive surgical therapy to regenerate interdental papillary soft tissue recession - a six-month postoperative image analysis-based clinical trial. J Korean Assoc Oral Maxillofac Surg 2021;47:438444. https://doi.org/10.5125/jkaoms.2021.47.6.438 